\title{
NUMERICAL EXPERIMENTS ON THE STABILITY OF LEADING EDGE BOUNDARY LAYER FLOW: A TWO- DIMENSIONAL LINEAR STUDY
}

\author{
VASSILIOS THEOFILIS* \\ Department of Applied Mathematics, University of Twente, Postbus 217, NL-7500 AE Enschede, Netherlands
}

\begin{abstract}
SUMMARY
A numerical study is performed in order to gain insight to the stability of the infinite swept attachment line boundary layer. The basic flow is taken to be of the Hiemenz class with an added cross-flow giving rise to a constant thickness boundary layer along the attachment line. The full Navier-Stokes equations are solved using an initial value problem approach after two-dimensional perturbations of varying amplitude are introduced into the basic flow.

A second-order-accurate finite difference scheme is used in the normal-to-the-wall direction, while a pseudospectral approach is employed in the other directions; temporally, an implicit Crank-Nicolson scheme is used. Extensive use of the efficient fast Fourier transform (FFT) algorithm has been made, resulting in substantial savings in computing cost.

Results for the two-dimensional linear regime of perturbations are in very good agreement with past numerical and theoretical investigations, without the need for specific assumptions used by the latter, thus establishing the generality of our method.
\end{abstract}

KFY wORDS Hydrodynamic stability Leading edge boundary layer

\section{INTRODUCTION}

Interest in studying leading edge contamination has recently intensified both in the U.S.A. and in Europe, the motivation stemming from the efforts to build laminar flow wings for transonic commercial use and also for hypersonic vehicle projects such as NASP (National Aerospace Plane) in the U.S.A. and HERMES and SÄNGER in Europe. ${ }^{1,2}$ A Direct Numerical Simulation (DNS) of flow in the Leading Edge Boundary Layer (LEBL) is presented aimed, within the limitations of the available computing resources, at addressing the problem posed by the still wide gap between theoretical/numerical investigations and experimental results while departing from normal mode analyses.

The infinite swept attachment line flow may be described by the classical stagnation point flow due to Hiemenz ${ }^{3}$ with an added spanwise component, assuming that the curvature of the region in the immediate vicinity of the attachment line may be neglected. This cross-flow is homogeneous along the attachment line and the resulting boundary layer is of constant thickness (in contrast to the Blasius profile). It is well known that the resulting flow may be represented by an exact solution of the Navier-Stokes equations. This feature is of particular importance for the

* Previous address: Department of Mathematics, University of Manchester, Manchester M13 9PL, U.K. 
stability analysis, since no boundary layer approximation is necessary and hence the search for a critical value of the Reynolds number for the onset of transition is based on a self-consistent theory (unlike the Orr-Sommerfeld analysis used in the Blasius boundary layer stability investigations).

Görtler ${ }^{4}$ and Hämmerlin ${ }^{5}$ originally studied this instability for the unswept $2 \mathrm{D}$ stagnation point case assuming that the chordwise component of the perturbation velocity $\tilde{u}$ depended linearly on the chordwise co-ordinate $x$, only quoting reasons of mathematical feasibility as justification for their assumption. All subsequent investigations adopted this or the extended form

$$
\tilde{u}=x u_{1}+x^{3} u_{3}+\ldots
$$

Many years later, Spalart, ${ }^{6}$ performing a direct simulation of the instability, turbulence and re-laminarization of flow on swept and unswept cylinders, observed the white noise perturbations introduced into the flow initially assuming the Görtler-Hämmerlin form after a long integration time. The theoretical works of Görtler and Hämmerlin, though, did not yield the crucial result (from an engineering point of view) of a critical Reynolds number for the onset of instability.

Theoretically, the investigation of the stability of the infinite swept attachment line flow continued with the work of Hall et al., ${ }^{7}$ who studied the linear regime of the disturbances using a normal mode analysis for the perturbations and solved the resulting eigenvalue problem, while Hall and Malik $^{8}$ extended this work into the weakly non-linear regime using analytical and numerical methods. From the first work it was concluded that the theoretical momentum thickness Reynolds number for the linear onset of instability in the attachment line boundary layer was $R_{\theta}=236$, while Hall and Malik $^{8}$ reported subcritical instability, quoting $R_{\theta} \approx 216$. Jiménez et al. ${ }^{9}$ also reported a value for $R_{\theta}$ consistent with the findings of Reference 7 , but were unable to find subcritical instability as in Reference 8.

Experiments were performed in order to assess the value of this important physical quantity. For a review of the pioneering work in leading edge contamination the reader is referred to Reference 1 . The main conclusions of the comprehensive experimental study of LEBL stability by Poll $^{10-14}$ were that the upper limit for the stability of the laminar swept attachment line boundary layer flow occurs at momentum thickness Reynolds number $R_{\theta} \approx 230$, also establishing that yaw of the cylinder has a powerful destabilizing effect at sufficiently high freestream Reynolds numbers. In the absence of instability at the attachment line, for a fixed yaw, the location of the transition moves progressively forward towards the attachment line as the freestream Reynolds number is increased. If the spanwise Reynolds number based on momentum thickness exceeds approximately 230 , though, instability occurs at the attachment line itself. This argument is invalid if large disturbances generated at the wing fuselage junction or at boundary layer fences are present; in this case turbulence occurs at the attachment line itself, even at $R_{\theta}$ as low as 100 .

Our contribution has been a numerical study of the stability of the linear regime of flow in the swept attachment line boundary layer. Previous investigations have been either direct simulations or solutions of eigenvalue problems resulting from a normal mode analysis of the perturbations introduced into the flow. Within memory and CPU limitations (working on a fixed budget on the Amdahl 1100 and 1200 supercomputers of Manchester Computing Centre) we chose the first course of action, since the second approach has received a fair amount of attention over the years by a number of investigators. The extent to which our approach, namely time marching as opposed to solution of an eigenvalue problem, may be applicable is investigated and results are obtained and compared with past investigations.

The reference point for this work of ours has been that of Hall et al. ${ }^{7}$ Theirs is a thorough investigation of the linear regime of perturbations using an eigenvalue approach. Extensive results have been presented, serving as a model to compare against and validate our initial- 
value-problem-based numerical scheme. Two direct numerical simulations also exist presently, namely those of Spalart ${ }^{6}$ and Jiménez et al. ${ }^{9}$ The objective of the former work has been a simulation of the full transition and rc-laminarization process in the LEBL, while the latter has been an investigation of the linear and non-linear regimes, both in incompressible flow. The numerical method of Spalart ${ }^{6}$ is fully spectral in space, while both the work of Jimenez et al. ${ }^{9}$ and ours use Fourier expansions in the periodic directions on the wall, along the attachment line and normal to it respectively, and finite differences in the direction normal to the wall.

Although using similar tools, the works of both Spalart ${ }^{6}$ and Jiménez et al. ${ }^{9}$ differ from ours in scope, ours being more focused on a specific flow regime. Our attempt is to present, using DNS and reasonable computing times, a detailed picture of LEBL instability while perturbations are still of small amplitude. The extent to which the eigenvalue approach adopted by Hall et al. ${ }^{7}$ and Hall and Malik ${ }^{8}$ is necessary for a description of linear instability in the LEBL is one of the aims of our investigation. On the basis of results presented in References 6 and 9, the only comparison possible between their work and ours is the consistency in the value of $R_{\theta}$ in the linear regime, all three simulations confirming the result of Reference 7 (and the persisting discrepancy between linear theory and experiment). We defer addressing the apparent contradiction between the results of References 8 and 9 regarding the existence of subcritical instability in the non-linear regime of perturbations to a later (non-linear) study. To this end we will first attempt to establish the applicability of our method in the linear regime.

\section{NUMERICAL SOLUTION PROCEDURE}

The first assumption that we make in order to solve for the base flow over the windward face of an infinite swept cylinder, as already discussed, is that this face may be treated as locally flat.* The oncoming flow outside the boundary layer is taken to be a stagnation point flow with a streamwise velocity component proportional to the distance from the leading edge. Taking $x$ to be the chordwise direction, $y$ to be the normal to the attachment line direction and $z$ to be the spanwise direction (along the attachment line), we define the basic flow by its non-dimensional components $(U, V, W)$ along the directions $(x, y, z)$ respectively. The dimensional lengths are $L(x, y, z)$, where $L$ is a typical length scale along the $x$-direction, and the respective dimensional velocities are

$$
W_{\mathrm{c}}\left(\frac{U}{R}, \frac{V}{R}, W\right) \text {, }
$$

where $W_{\mathrm{c}}$ is the $z$-velocity component of the freestream velocity $\left(U_{\mathrm{e}}, V_{\mathrm{c}}, W_{\mathrm{e}}\right)$ sufficiently far from the surface. $R$ is the Reynolds number of the flow,

$$
R=\frac{W_{\mathrm{e}} \Delta}{v}
$$

based on $\Delta$, the boundary layer length scale,

$$
\Delta=\left(\frac{v L}{U_{\mathrm{c}}}\right)^{1 / 2},
$$

and $v$ is the kinematic viscosity of the flow. In the absence of any disturbance the basic flow is

* Terms $O\left(\kappa R_{L}^{-1 / 2}\right)$ and $O\left(R_{L}{ }^{3 / 2} \partial \kappa / \partial x\right), \kappa$ being the curvature of the wall and $R_{L}$ the Reynolds number based on an $O(1)$ length $L$, are introduced into the Navier -Stokes equations when the equations are written for flow past a cylindrical body including curvature. ${ }^{15}$ In view of the largeness of $R_{L}$ in this work, these terms will be neglected. 
taken to be of the form

$$
U=\frac{x}{R} \bar{u}(y), \quad V=\frac{1}{R} \bar{v}(y), \quad W=\bar{w}(y),
$$

which, if substituted into the Navier-Stokes equations, leads to the system of ordinary differential equations of the Falkner-Skan family,

$$
\begin{aligned}
\bar{u}+\bar{v}^{\prime} & =0, \\
\bar{v}^{\prime \prime \prime}+\bar{v}^{\prime 2}-\bar{v} \bar{v}^{\prime \prime}-1 & =0, \\
\bar{w}^{\prime \prime}-\bar{v} \bar{w}^{\prime} & =0,
\end{aligned}
$$

with boundary conditions

$$
\begin{aligned}
& \vec{v}(0)=\kappa, \quad \bar{v}^{\prime}(0)=0, \quad \bar{v}^{\prime}(\infty)=-1, \\
& \bar{w}(0)=0, \quad \bar{w}(\infty)=1,
\end{aligned}
$$

where prime denotes differentiation with respect to the similarity variable $\eta=y / \Delta$ and $k$ is a nondimensional parameter used to control blowing/suction in the boundary layer. This reduced form of the Navier-Stokes equations represents a generalization of the well-known Hiemenz ${ }^{3}$ flow in that, in addition to the plane stagnation point flow, it incorporates a non-zero spanwise velocity component. A straightforward shooting technique is used to solve this problem in order to obtain the basic flow velocity profile. Details of the solution may be found in References 7, 8 and 16-20 among others.

In formulating the perturbed flow problem, we shall adopt the velocity-vorticity formulation for the Navier-Stokes equations as described for example in Reference 21. This method was chosen firstly to avoid ambiguities in the specification of boundary conditions for the pressure at the wall and secondly because it is readily applicable to three-dimensional situations. The latter, i.e. a study of the three-dimensional regime of perturbations, is one of the potential extensions of the present work. Using this approach and considering the flow to be incompressible, the threedimensional unsteady Navier-Stokes equations take the (non-dimensionalized) form

$$
\begin{aligned}
& \nabla^{2} \tilde{u}=-\nabla \times \tilde{\zeta}, \\
& \nabla^{2} \tilde{\zeta}=R\left(\frac{\partial \tilde{\zeta}}{\partial t}+(\tilde{u} \cdot \nabla) \tilde{\zeta}-(\tilde{\zeta} \cdot \nabla) \tilde{u}\right),
\end{aligned}
$$

where $\tilde{u}=(u, v, w)$ is the velocity vector and $\tilde{\zeta}=(\xi, \eta, \zeta)$ is the vorticity.

Small perturbations are introduced in the flow over the swept cylinder. The velocity field describing the flow may then be taken to be a superposition of the basic flow terms and perturbation velocities. Two-dimensional forms of the perturbation velocities will be assumed. These velocities and the corresponding vorticities are substituted into the basic set of equations (4) and (5) and the Hiemenz flow is subtracted. A pseudospectral method is employed to calculate the spatial derivatives in the spanwise direction $z$ and the resulting equations are discretized in spectral space using a second-order finite difference scheme in the normal direction $y$.

The impetus to the implementation of spectral methods was given by the efficient algorithm of Cooley and Tuckey, ${ }^{22}$ namely the fast Fourier transform (FFT) algorithm. We implemented the spectral idea using an approach similar (in spirit, if not in detail) to that of References 23-25, to which the reader is referred for details regarding the precise definition of the Fourier transform implemented as well as the discretization scheme in both physical and transform spaces. 
A (second-order) Crank-Nicolson ${ }^{26}$ scheme is used for the time marching of the equations, an implicit scheme chosen because of its superior stability properties over explicit schemes. The resulting equations to be solved take the form of a system of simultaneous algebraic equations, written symbolically as

$$
\mathbf{A X}=\mathbf{B}
$$

at constant wave number and time. The solution of this system (only practically feasible owing to the banded structure of $\mathbf{A}$ ) may be used to update the flow quantities at the new time level.

Having obtained the solution at a given time level in spectral (i.e. Fourier) space in the general form $f^{*}(y, \beta)$ ( $\beta$ being the spanwise wave number), we back-transform into real space, thus obtaining the velocity, vorticity and other quantities of interest in physical space in the general form $f(y, z)$, employing the FFT method referred to earlier. Since real space quantities are real, their spectral counterparts are Hermitian sequences and this results in significant savings in computer storage and time as the grid requirements increase. The triggering of the (unsteady) set of equations comes from forcing the normal component of the perturbation velocity on $y=0$, thus modelling physically attainable situations and giving our method potential for direct comparison with experimental data.

\section{A TWO-DIMENSIONAL, LINEAR, TIME-MARCHING SCHEME}

\subsection{Assumptions and governing equations}

The assumptions made here are: firstly, the basic flow is taken to be the generalized Hiemenz flow which we solved for earlier; secondly, the perturbation velocities are taken to be functions of $(y, z, t)$, where $y$ is the normal to the surface co-ordinate, $z$ is the spanwise co-ordinate and $t$ is time (a fact stemming from the two-dimensionality of the flow in the attachment line boundary layer); thirdly, the chordwise velocity components of basic and perturbed flow are linearly dependent on the chordwise co-ordinate $x$.

If $(\bar{u}, \bar{v}, \bar{w})$ is the velocity vector describing the basic flow and $(\tilde{u}, \tilde{v}, \tilde{w})$ is the vector describing the perturbed flow, then we assume

$$
\begin{array}{ll}
\bar{u}=x U(y), & \tilde{u}=x \hat{u}(y, z, t), \\
\bar{v}=V(y), & \tilde{v}=\hat{v}(y, z, t), \\
\bar{w}=W(y), & \tilde{w}=\hat{w}(y, z, t),
\end{array}
$$

where $(U, V, W)$ is the velocity vector describing the Hiemenz flow and $(\hat{u}, \hat{v}, \hat{w})$ is the perturbation velocity. It follows for the vorticity that

$$
\begin{array}{ll}
\bar{\xi}=W^{\prime}(y), & \tilde{\xi}=\tilde{w}_{y}-\tilde{v}_{z}=\hat{\xi}(y, z, t), \\
\bar{\eta}=0, & \tilde{\eta}=x \tilde{u}_{z}=x \hat{\eta}(y, z, t), \\
\bar{\zeta}=-x U^{\prime}(y), & \tilde{\zeta}=-x \tilde{u}_{y}=-x \hat{\zeta}(y, z, t),
\end{array}
$$

with bars referring to basic flow quantities and hats and tildes referring to the respective perturbations; prime denotes differentiation with respect to $y$ and subscripts denote partial differentiation with respect to the corresponding spatial co-ordinate. Substituting these forms into the basic set of equations (4) and (5) and Fourier transforming leads to the following set of disturbance equations in spectral space (having subtracted the basic Hiemenz-flow-related 
terms):

$$
\begin{gathered}
\frac{\partial^{2} \hat{u}^{*}}{\partial y^{2}}-\beta^{2} \hat{u}^{*}-\mathrm{i} \beta \hat{\eta}^{*}-\frac{\partial \hat{\zeta}^{*}}{\partial y}=0 \\
\frac{\partial^{2} \hat{v}^{*}}{\partial y^{2}}-\beta^{2} \hat{v}^{*}+\mathrm{i} \beta \hat{\xi}^{*}+\hat{\zeta}^{*}=0 \\
\frac{\partial^{2} \hat{w}^{*}}{\partial y^{2}}-\beta^{2} \hat{w}^{*}+\hat{\eta}^{*}-\frac{\partial \hat{\xi} *}{\partial y}=0 \\
R^{-1}\left(\frac{\partial^{2} \hat{\xi}^{*}}{\partial y^{2}}-\beta^{2} \hat{\zeta}^{*}\right)-\frac{\partial \hat{\xi}^{*}}{\partial t}-V \frac{\partial \hat{\xi}^{*}}{\partial y}-\hat{v}^{*} \frac{\mathrm{d} \bar{\xi}}{\mathrm{d} y}-\mathrm{i} \beta W \hat{\xi}^{*}+U \hat{\xi}^{*}+\bar{\xi} \hat{u}^{*}=(\operatorname{RHS} 1)^{*} \\
R^{-1}\left(\frac{\partial^{2} \hat{\eta}^{*}}{\partial y^{2}}-\beta^{2} \hat{\eta}^{*}\right)-\frac{\partial \hat{\eta}^{*}}{\partial t}-V \frac{\partial \hat{\eta}^{*}}{\partial y}+\hat{\eta}^{*} \frac{\mathrm{d} V}{\mathrm{~d} y}-\mathrm{i} \beta W \hat{\eta}^{*}-U \hat{\eta}^{*}-\mathrm{i} \beta W \hat{\zeta}^{*}=(\operatorname{RHS} 2)^{*} \\
\left.\partial y^{2}-\beta^{2} \hat{\zeta}^{*}\right)-\frac{\partial \hat{\zeta}^{*}}{\partial t}-V \frac{\partial \hat{\zeta}^{*}}{\partial y}-\hat{v}^{*} \frac{\mathrm{d} \bar{\zeta}}{\mathrm{d} y}-\mathrm{i} \beta W \hat{\zeta}^{*}-U \hat{\zeta}^{*}-\bar{\zeta} \hat{u}^{*}+\mathrm{i} \beta \bar{\zeta} \hat{w}^{*}-\frac{\mathrm{d} W}{\mathrm{~d} y} \hat{\eta} \hat{\eta}^{*}=(\operatorname{RHS} 3)^{*},
\end{gathered}
$$

where asterisks denote spectral (Fourier) space functions and RHS1-RHS3 denote the quadraticin-perturbation right-hand sides of the equations, which in this linear scheme are set to zero. No-slip boundary conditions for the $x$ - and $z$-components of the velocity are imposed and the definition of vorticity is used to derive boundary conditions for the vorticity, namely

$$
\begin{aligned}
& \hat{\xi}^{*}(y=0)=\hat{w}_{y}^{*}(0)-\mathrm{i} \beta \hat{v}^{*}(0), \\
& \hat{\eta}^{*}(y=0)=0, \\
& \zeta^{*}(y=0)=\hat{u}_{y}^{*}(0),
\end{aligned}
$$

with subscripts denoting partial differentiation and derivatives at the wall calculated using standard one-sided, second-order-accurate finite difference formulae. In the far field we expect all perturbations to vanish:

$$
\begin{array}{ll}
\hat{u}^{*}(y \rightarrow \infty) \rightarrow 0, & \hat{\xi}^{*}(y \rightarrow \infty) \rightarrow 0, \\
\hat{v}^{*}(y \rightarrow \infty) \rightarrow 0, & \hat{\eta}^{*}(y \rightarrow \infty) \rightarrow 0, \\
\hat{w}^{*}(y \rightarrow \infty) \rightarrow 0, & \hat{\zeta}^{*}(y \rightarrow \infty) \rightarrow 0 .
\end{array}
$$

A number of different cases were considered for the function $\hat{v}^{*}(0)$ which acts as the flow forcing. A universal requirement for the forcing, for all schemes utilized, was that the function should be bounded everywhere, both in time and in real and spectral space. In the case where the physical situation which we wanted to model was an impulse (corresponding, for example, to a jet impinging from the surface normal to the wall), the forcing in spectral space was chosen to be a short-lived, smoothly growing and decaying (temporally and spatially) function such as

$$
\hat{v}^{*}(y=0, \beta, t)=h f(t) \tanh ^{2}(t)\left(1-\tanh ^{2}(t)\right) \mathrm{e}^{-\beta^{2}} .
$$

If, on the other hand, we modelled the situation where waves in the boundary layer are set up by a periodic physical forcing, such as a vibrating ribbon, a (temporal) sinusoidal form of the forcing function was considered. In spectral space this was

$$
\hat{v}^{*}(y=0, \beta, t)=h f(t) \sin (t) \mathrm{e}^{-\beta^{2}} .
$$


In both cases $h$ is an $O(1)$ amplitude function used to control the magnitude of the forcing applied to the normal component of the velocity. By increasing $h$, we expect to enter the regime where non-linear effects become progressively more important. The unit step function

$$
f(t)= \begin{cases}0, & t<0, \\ 1, & t \geqslant 0\end{cases}
$$

is used to indicate the finite start-up time in our initial value problem calculations. Other, more general forms of perturbations might have been studied in place of the error function type of disturbance in (13) and (14). They should also be bounded in space and time. However, in view of the need for special treatment in spectral space in order to overcome potentially arising slow convergence when a general form of disturbance is used, we chose to use the error function type, expecting no loss of generality in capturing the important physics by this simpler form.

\subsection{Time-marching numerical solution}

Considering the discretization scheme discussed earlier when applied to (7) (12), the meaning of the matrices in (6) now becomes clear. $\mathbf{A}$ is a $6 N y \times 6 N y$ banded matrix with bandwidth determined by the number of unknowns and the order of the finite difference scheme used. $N y$ is the number of points in the $y$-direction. In the case solved, we have six unknowns, i.e. three velocity and three vorticity components, and the second-order finite difference scheme used requires functions at three neighbouring grid points; hence the bandwidth of $\mathbf{A}$ is 18 (block tridiagonal).

$\mathrm{X}$ is a column vector of length $6 \mathrm{Ny}$, each entry of which is a velocity or vorticity component on a $y$-grid point at the same (current) time level.

$\mathrm{B}$ is also a column vector of length $6 N y$ containing the right-hand sides of the equations, velocity and vorticity components at the previous time level, as well as the boundary conditions.

We first determine the Hiemenz flow solution and normalize the $x$ - and $y$-velocities and their derivatives by the Reynolds number $R$, consistent with the assumptions made earlier. We then set up the coefficients of $\mathbf{A}$. The six solution components, i.e. three velocities and three vorticities, are only stored at two time levels, namely the previous and the current. Initially we set all solution components equal to zero and expect the solution to be driven by the wall forcing. The system of equations (6) is set up and solved by Gaussian elimination using a set of $\mathrm{NAG}^{27}$ library subroutines appropriate for vector machines. The solution consists of the three velocities and three vorticities in spectral space at the current time level. Having obtained the solution in spectral space, we overwrite the previous time level solution with the current time level solution and solve for the next time step. The solution in real space has to be calculated at each time step and this is also carried out by NAG library subroutines which efficiently perform FFTs on vector machines.

In order to assess the consistency and accuracy of the solution, an extensive grid experimentation was performed. Different values of the time step parameter as well as the number of grids in both the (uniform) normal $(y)$ and spanwise $(z)$ directions were tested. Within the limitations presented by the machines used, it was concluded ${ }^{20}$ that the choice of

$$
N y=1251 \times N z=40 \text { with } \Delta \beta=0 \cdot 125
$$

is the optimal compromise between the desire for as fine a resolution as possible and memory restrictions. 


\subsection{Results}

Perturbation velocity and vorticity profiles at the midpoint of the $z$-range $(z=0)$, results typical of those obtained in Reference 20, are presented in Figures 1-3. The sinusoidal forcing (14) was used and $t=10 \pi$ (when the transient behaviour of the solution has subsided).

In order to validate the present method, we compare our results with those of Hall et al. ${ }^{7}$ They investigated the linear regime of perturbations introduced in the basic flow using a temporal approach and solved the respective eigenvalue problem, thus establishing the critical Reynolds number for the onset of instability as well as the effects of wall suction/blowing on stability.

Using the wall forcing function (in spectral space)

$$
\hat{v}^{*}(y=0, \beta, t)=h f(t) \tanh ^{2}(t)\left(1-\tanh ^{2}(t)\right) \mathrm{e}^{-\beta^{2}}
$$

and the time step $\Delta t=0 \cdot 1$, we solved for just one value of the spanwise wave number $\beta$ at a time and observed the development of any flow quantity with time. In $(R, \beta)$ space we varied $R$ and $\beta$, marched the solution in time and observed whether damped or amplified oscillations occur; if $(R, \beta)$ is located inside the neutral curve predicted by Reference 7, we expect temporally growing oscillations to be observed, while if the $(R, \beta)$ point falls outside the neutral loop, the temporal oscillations observed should be damped. The choice of a pair of parameters on the neutral loop of Reference 7 should produce oscillations of constant amplitude.

We ran a number of $(R, \beta)$ cases; typical results obtained are given in Table $I$, indicating the status (growing or decaying) that a perturbation introduced into the flow is found in for a specific

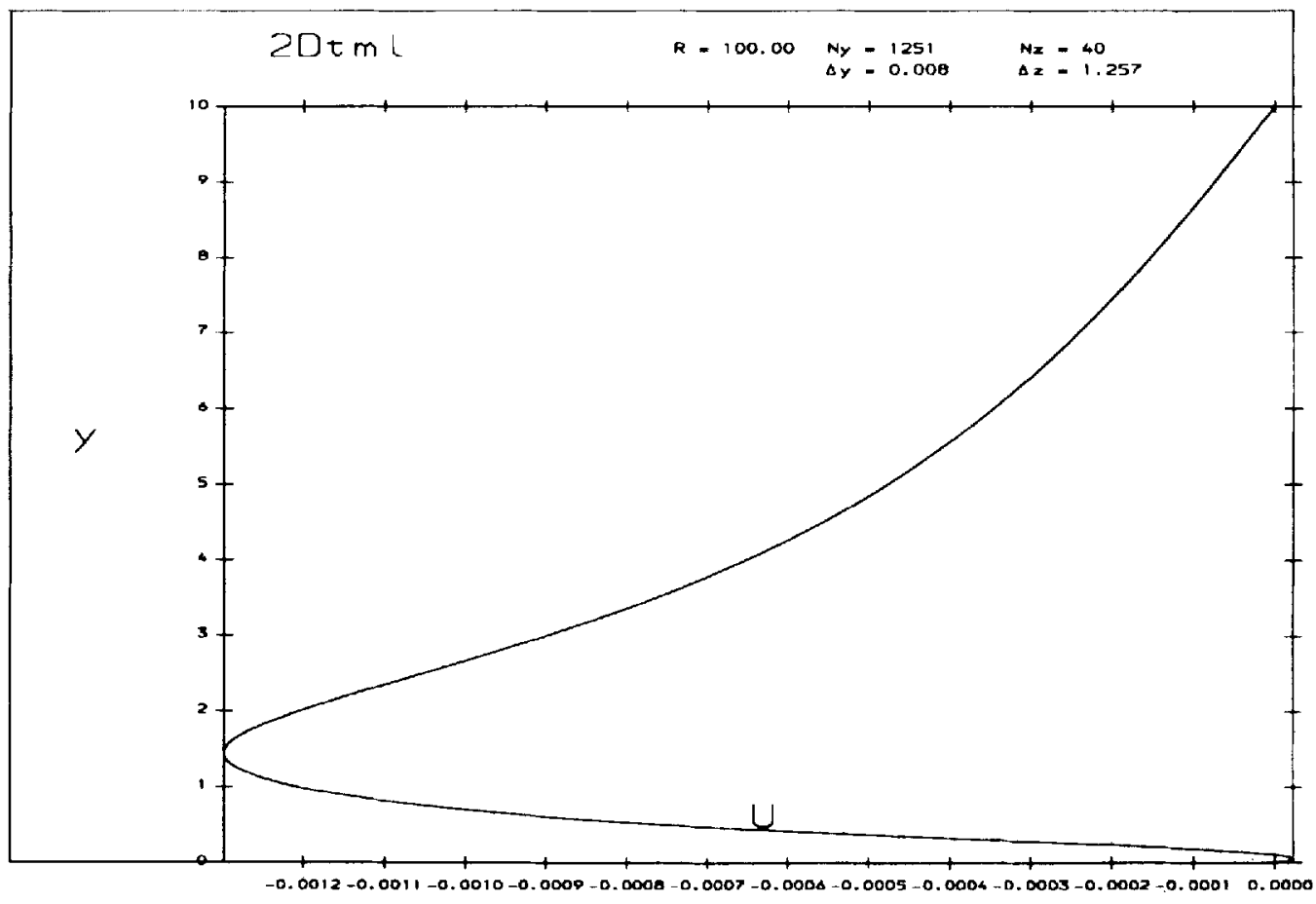

Figure 1. Time-marching results: perturbation velocity profile $\hat{u}(z=0)$ 


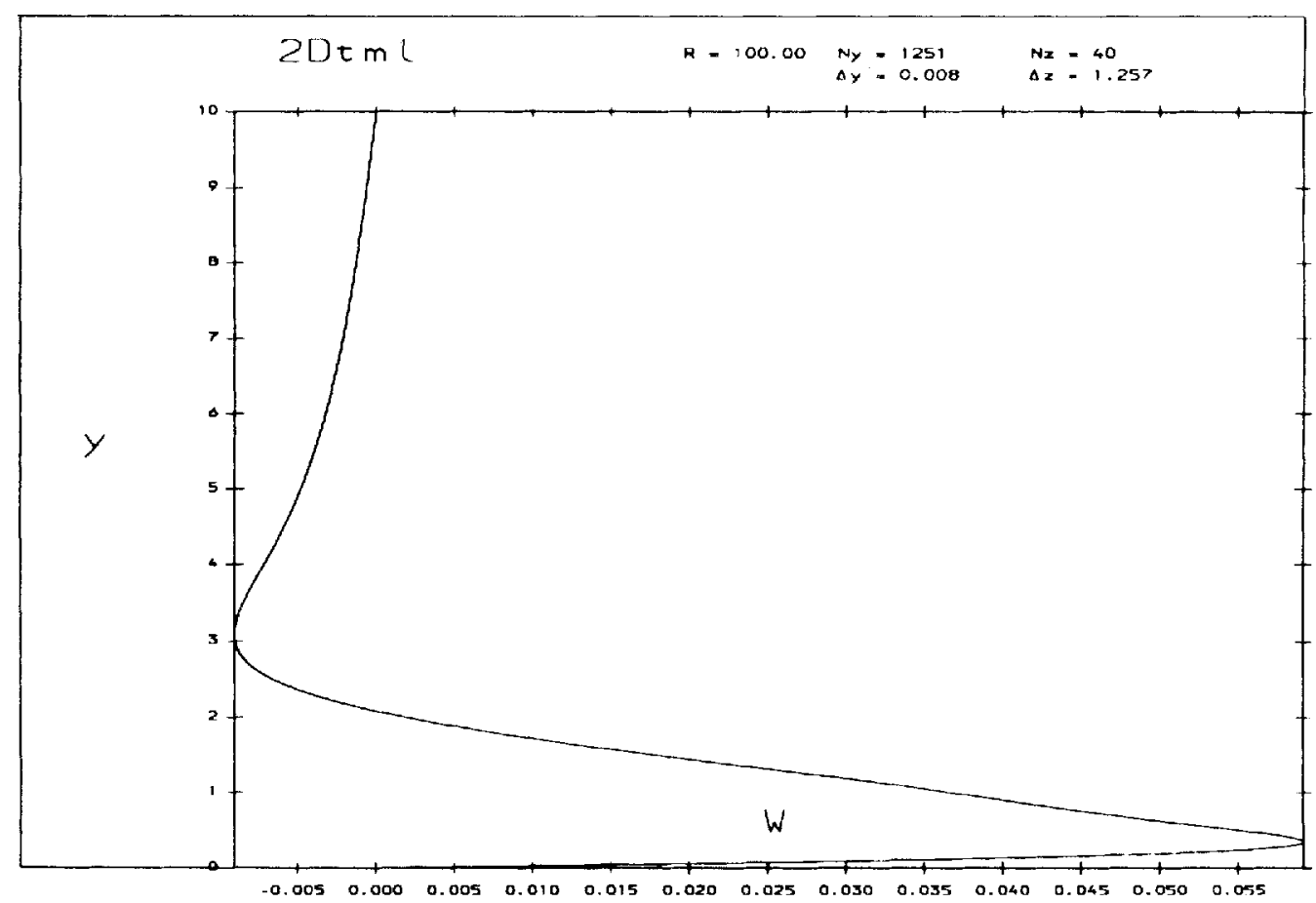

Figure 2. Time-marching results: perturbation velocity profile $\hat{w}(z=0)$

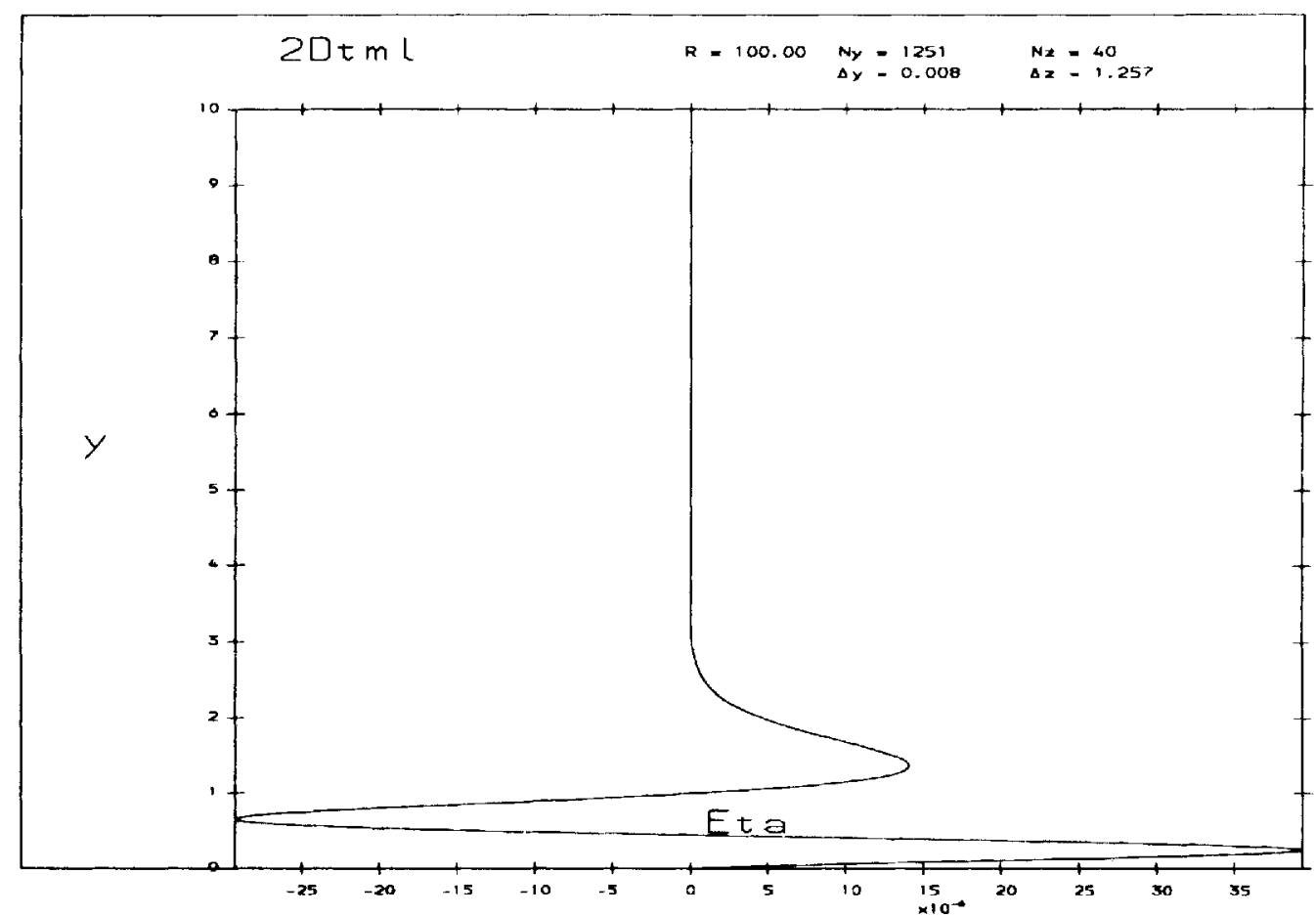

Figure 3. Time-marching results: perturbation vorticity profile $\hat{\eta}(z=0)$ 
Table I. Two-dimensional linear stability results $(N y=1001 \times N z=1)$

\begin{tabular}{lcccccr}
\hline$R$ & $\beta$ & Status & $\omega_{\mathrm{r}}$ & $f_{\mathrm{r}}=1 / \omega_{\mathrm{r}}$ & \multicolumn{1}{c}{$\Omega_{\mathrm{r}}$} & \multicolumn{1}{c}{$\Omega_{\mathrm{r}}$} \\
\hline 500.0 & 0.300 & $\mathrm{~d}$ & $52 \cdot 65 \pm 0 \cdot 05$ & $0 \cdot 01899 \pm 0 \cdot 00002$ & $-0 \cdot 11936 \pm 0 \cdot 00001$ & $0 \cdot 00049 \pm 0 \cdot 00001$ \\
$600 \cdot 0$ & $0 \cdot 300$ & $\mathrm{~g}$ & $54 \cdot 00 \pm 0 \cdot 10$ & $0 \cdot 01850 \pm 0 \cdot 00002$ & $-0 \cdot 11624 \pm 0 \cdot 00001$ & $-0 \cdot 00075 \pm 0 \cdot 00001$ \\
$820 \cdot 0$ & $0 \cdot 200$ & $\mathrm{~g}$ & $88 \cdot 69 \pm 0 \cdot 01$ & $0 \cdot 01127 \pm 0 \cdot 00001$ & $-0 \cdot 07085 \pm 0 \cdot 00001$ & $-0 \cdot 00083 \pm 0 \cdot 00002$ \\
$900 \cdot 0$ & $0 \cdot 350$ & $\mathrm{~g}$ & $48 \cdot 30 \pm 0 \cdot 01$ & $0 \cdot 02068 \pm 0 \cdot 00002$ & $-0 \cdot 13005 \pm 0 \cdot 00001$ & $-0 \cdot 00043 \pm 0 \cdot 00001$ \\
$700 \cdot 0$ & $0 \cdot 350$ & $\mathrm{~g}$ & $46 \cdot 50 \pm 0 \cdot 10$ & $0 \cdot 02148 \pm 0 \cdot 00003$ & $-0 \cdot 13503 \pm 0 \cdot 00001$ & $-0 \cdot 00012 \pm 0 \cdot 00001$ \\
$900 \cdot 0$ & $0 \cdot 375$ & $\mathrm{~d}$ & $44 \cdot 80 \pm 0 \cdot 10$ & $0 \cdot 02232 \pm 0 \cdot 00001$ & $-0 \cdot 14022 \pm 0 \cdot 00001$ & $0 \cdot 00143 \pm 0 \cdot 00001$ \\
$700 \cdot 0$ & $0 \cdot 375$ & $\mathrm{~d}$ & $43 \cdot 10 \pm 0 \cdot 10$ & $0 \cdot 02320 \pm 0 \cdot 00005$ & $-0 \cdot 14569 \pm 0 \cdot 00001$ & $0 \cdot 00133 \pm 0 \cdot 00001$ \\
$700 \cdot 0$ & $0 \cdot 200$ & $\mathrm{~d}$ & $86 \cdot 55 \pm 0 \cdot 05$ & $0 \cdot 01155 \pm 0 \cdot 00001$ & $-0 \cdot 07259 \pm 0 \cdot 00001$ & $0 \cdot 00030 \pm 0 \cdot 00001$ \\
$700 \cdot 0$ & $0 \cdot 225$ & $\mathrm{~g}$ & $76 \cdot 20 \pm 0 \cdot 10$ & $0 \cdot 01312 \pm 0 \cdot 00001$ & $-0 \cdot 08246 \pm 0 \cdot 00001$ & $-0 \cdot 00066 \pm 0 \cdot 00001$ \\
$900 \cdot 0$ & $0 \cdot 200$ & $\mathrm{~g}$ & $90 \cdot 00 \pm 0 \cdot 10$ & $0 \cdot 01111 \pm 0 \cdot 00001$ & $-0 \cdot 06984 \pm 0 \cdot 00001$ & $-0 \cdot 00141 \pm 0 \cdot 00001$ \\
$550 \cdot 0$ & $0 \cdot 300$ & $\mathrm{~g}$ & $53 \cdot 35 \pm 0 \cdot 05$ & $0 \cdot 01860 \pm 0 \cdot 00010$ & $-0 \cdot 11773 \pm 0 \cdot 00001$ & $-0 \cdot 00020 \pm 0 \cdot 00001$ \\
$900 \cdot 0$ & $0 \cdot 175$ & $\mathrm{~g}$ & $103 \cdot 60 \pm 0 \cdot 10$ & $0 \cdot 00965 \pm 0 \cdot 00001$ & $-0 \cdot 06065 \pm 0 \cdot 00001$ & $-0 \cdot 00036 \pm 0 \cdot 00001$ \\
\hline
\end{tabular}

choice of parameters $(R, \beta)$. The period of the oscillations observed is denoted ${ }^{*}$ by $\omega_{\mathrm{r}}$ and its inverse by $f_{\mathrm{r}}$. Growth rates calculated from

$$
\Omega=\frac{\ln \left(\hat{u}_{y}^{*}(t)\right)-\ln \left(\hat{u}_{y}^{*}(t-\Delta t)\right)}{i \Delta t}
$$

are also presented. $f$ The solution was computed until the growth rates had settled to the presented values (accurate to five decimal places); owing to the smallness of the imaginary parts of the growth rates, this was only achieved after (non-dimensional) time $t \approx 750$ had elapsed. A large number of different $(R, \beta)$ choices was run, of which typical results are presented in Figures 4-9, each representative of a case of growing and decaying oscillation of a flow quantity (in the case presented, a wall shear) and the corresponding growth rates.

Using our two-dimensional linear results, we were able to reproduce the neutral loop corresponding to that of Hall et al., ${ }^{7}$ which is presented in Figure 10 (from Reference 19) for two values of the non-dimensional suction parameter, $\kappa=0$ (no suction) and $\kappa=0.4$ (blowing). The critical Reynolds number $R_{\text {crit }}$ was found to be (as may well be expected) a function of the grid used. Bearing in mind the cost of the calculations for a full set of results in $(R, \beta)$ space, we used a fairly moderate number of points in the normal direction $(N y=1001)$ and this influenced the precise value of $R_{\text {crit }}$. Nevertheless, the agreement between the results obtained by the eigenvalue problem approach of Hall et $\mathrm{al}^{7}{ }^{7}$ and our calculations can be seen as quite satisfactory. A discrepancy at the lower branch of the neutral curve is attributed to insufficient resolution in the normal direction in our numerical solution and may well be expected, since a more accurate description of lower-branch instability would require some form of asymptotic analysis using triple-deck theory. The powerful destabilizing effect of blowing is also established.

Overall, the results build confidence in extending our method in order to study non-linear perturbations introduced in the infinite swept attachment line boundary layer flow, as well as the stability of the flow at stations off the attachment line. Before we turn our attention to the

\footnotetext{
* In the notation of Reference 7 the wave period is denoted by $\omega_{\mathrm{r}}$ and we have kept the same notation; the corresponding frequency in the notation of Reference 7 is $x c_{\mathrm{r}}$, corresponding to $f_{\mathrm{r}}$ in our notation. The oscillation period $(\omega)=\omega_{\mathrm{r}}+\mathrm{i} \omega_{i}$ should not be confused with the growth rate denoted by $\Omega=\Omega_{\mathrm{r}}+\mathrm{i} \Omega_{i}$, the relation between the real parts thereof being $\Omega_{\mathrm{r}}=2 \pi f_{\mathrm{r}}$.

$\dagger$ Identical results were obtained using the (standard) definition $(1 / A) \mathrm{d} A / \mathrm{d} t, A=\hat{u}_{y}^{*}(t)$.
} 


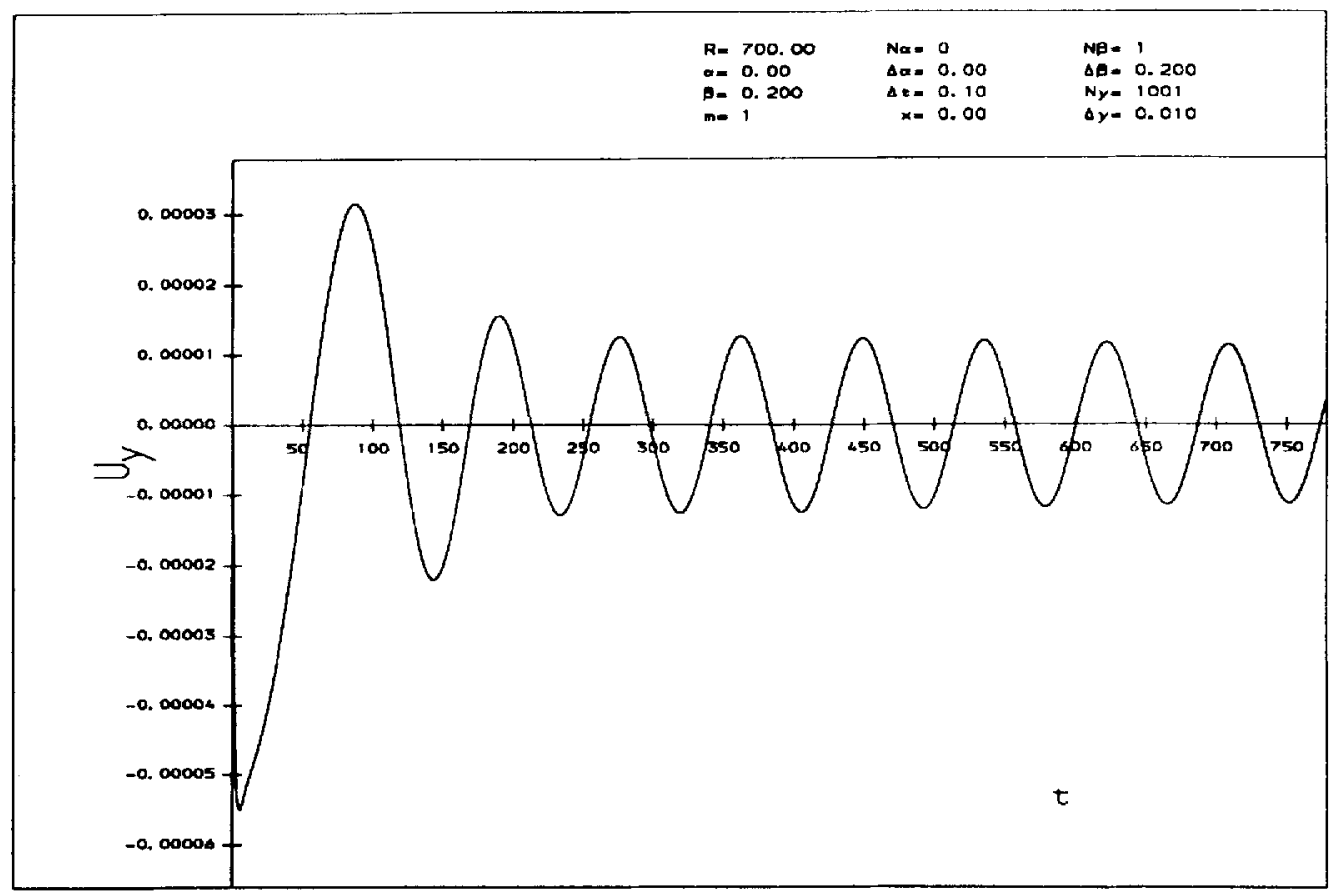

Figure 4. Time-marching results: wall shear $\hat{u}_{y}$ versus $t ; R=700, \beta=0.2$

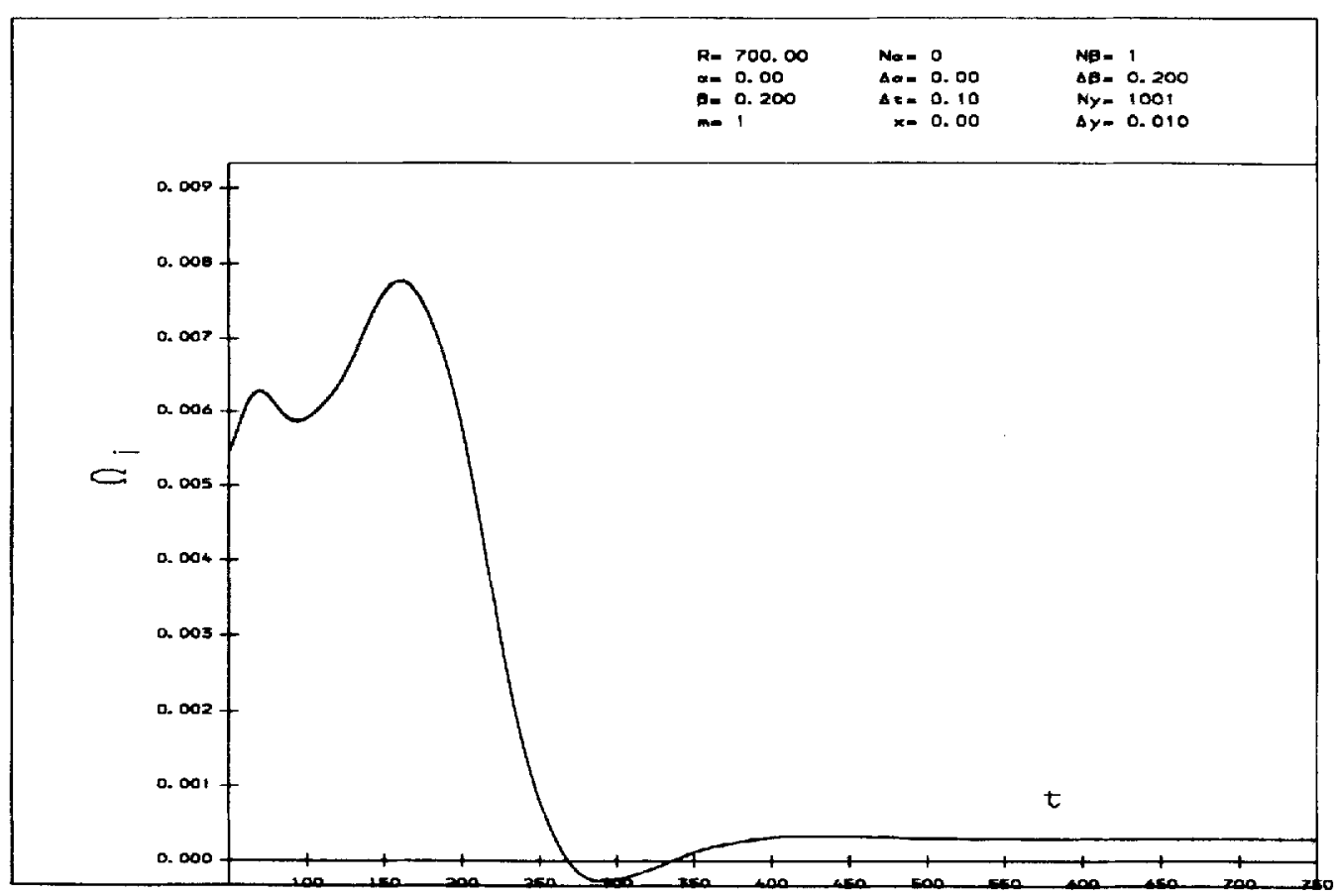

Figure 5. Time-marching results: growth rate $\Omega_{i}$ versus $t ; R=700, \beta=0 \cdot 2$ 


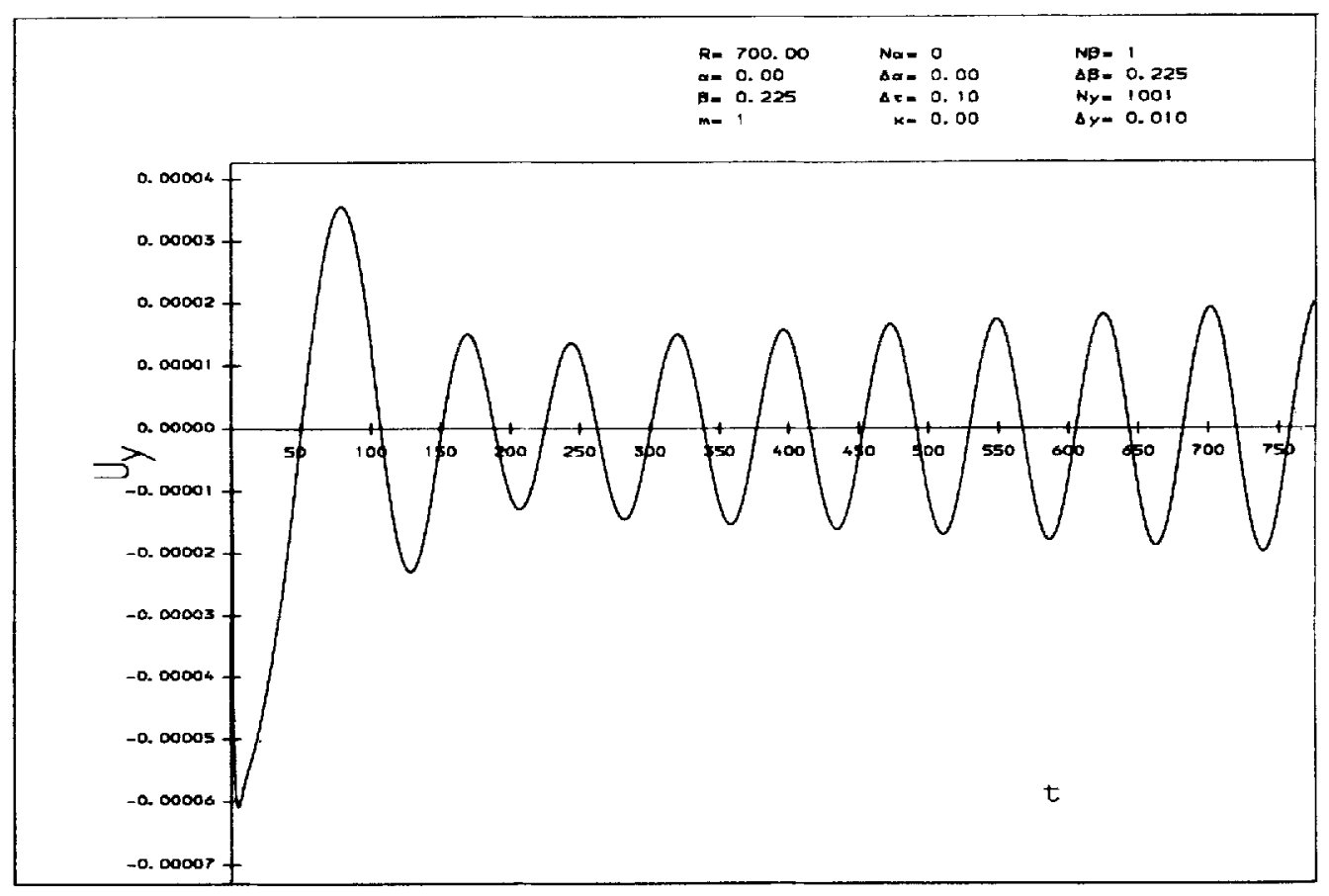

Figure 6. Time-marching results: wall shear $\hat{u}_{y}$ versus $t ; R=700, \beta=0.225$

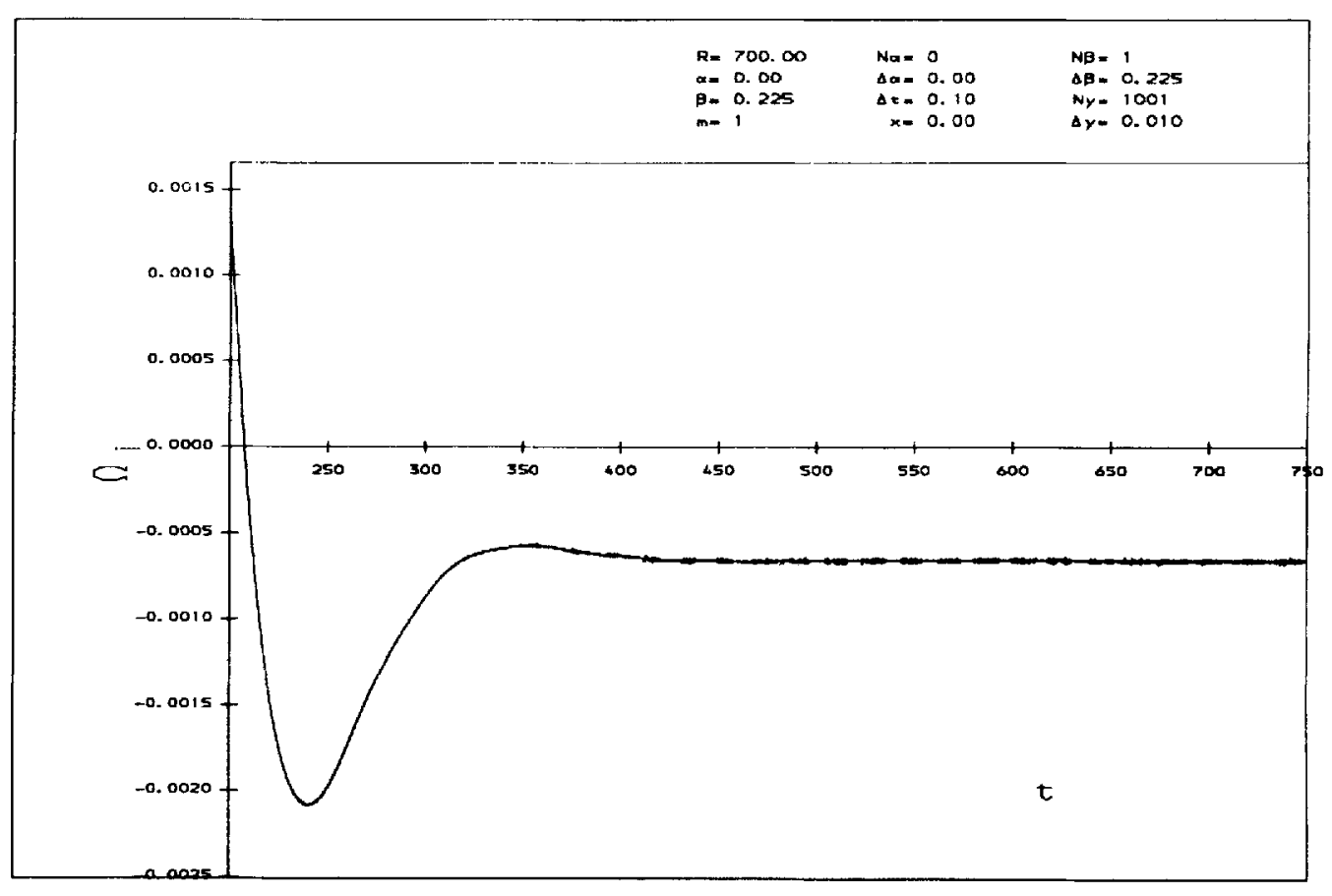

Figure 7. Time-marching results: growth rate $\Omega_{i}$ versus $t ; R=700, \beta=0.225$ 


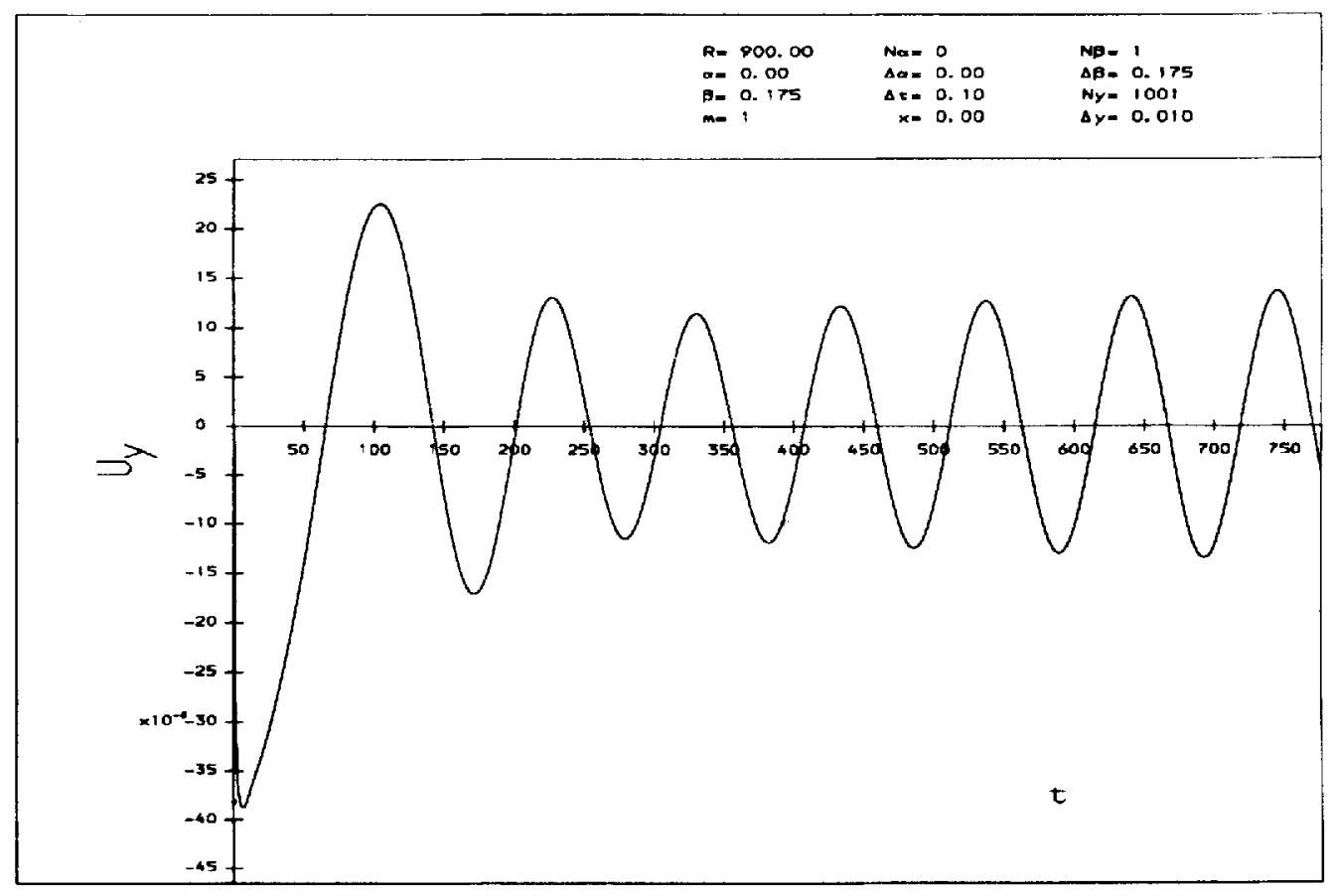

Figure 8. Time-marching results: wall shear $\hat{u}_{y}$ versus $t ; R=900, \beta=0-175$

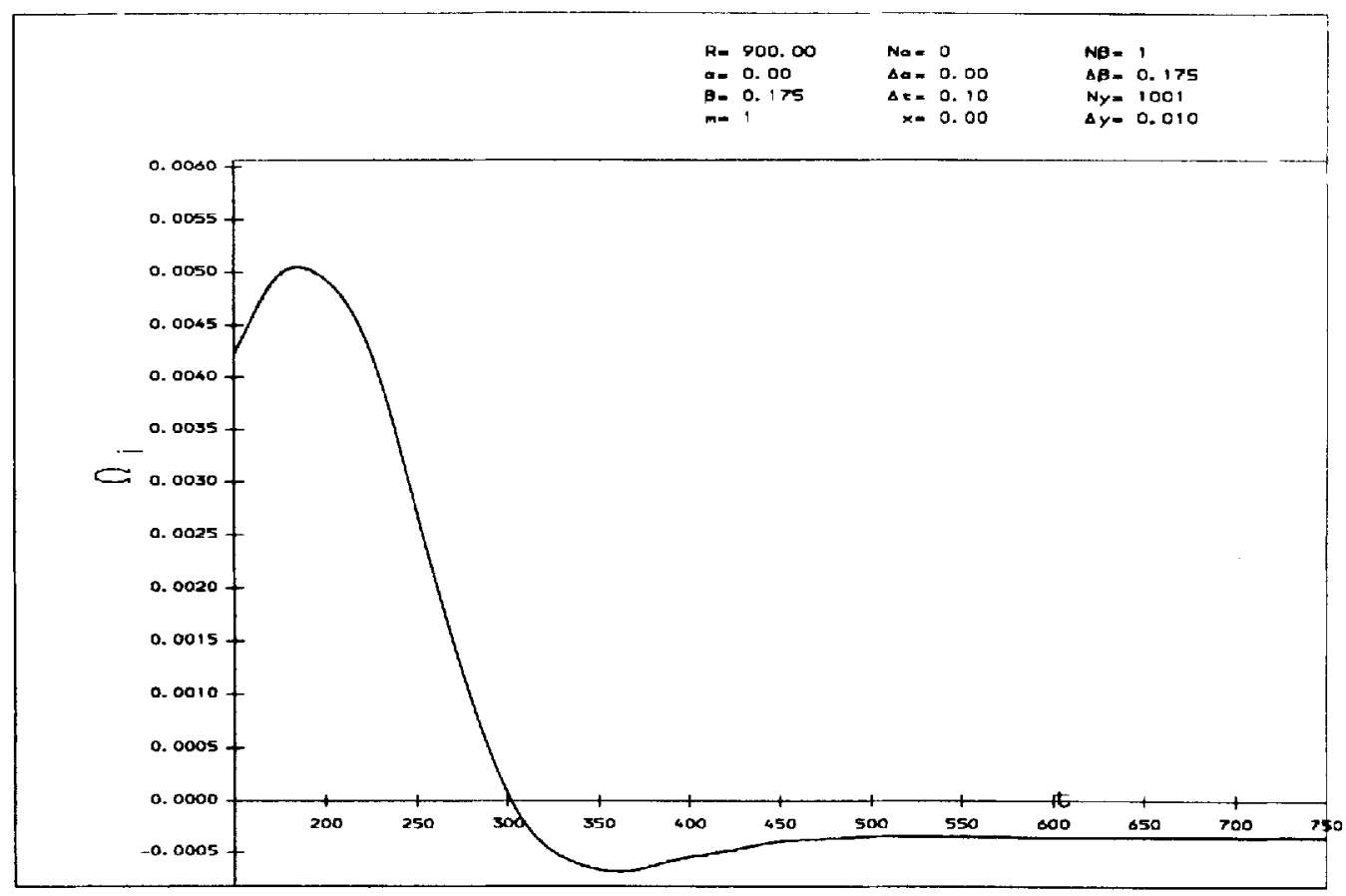

Figure 9. Time-marching results: growth rate $\Omega_{i}$ versus $t ; R=900, \beta=0 \cdot 175$ 


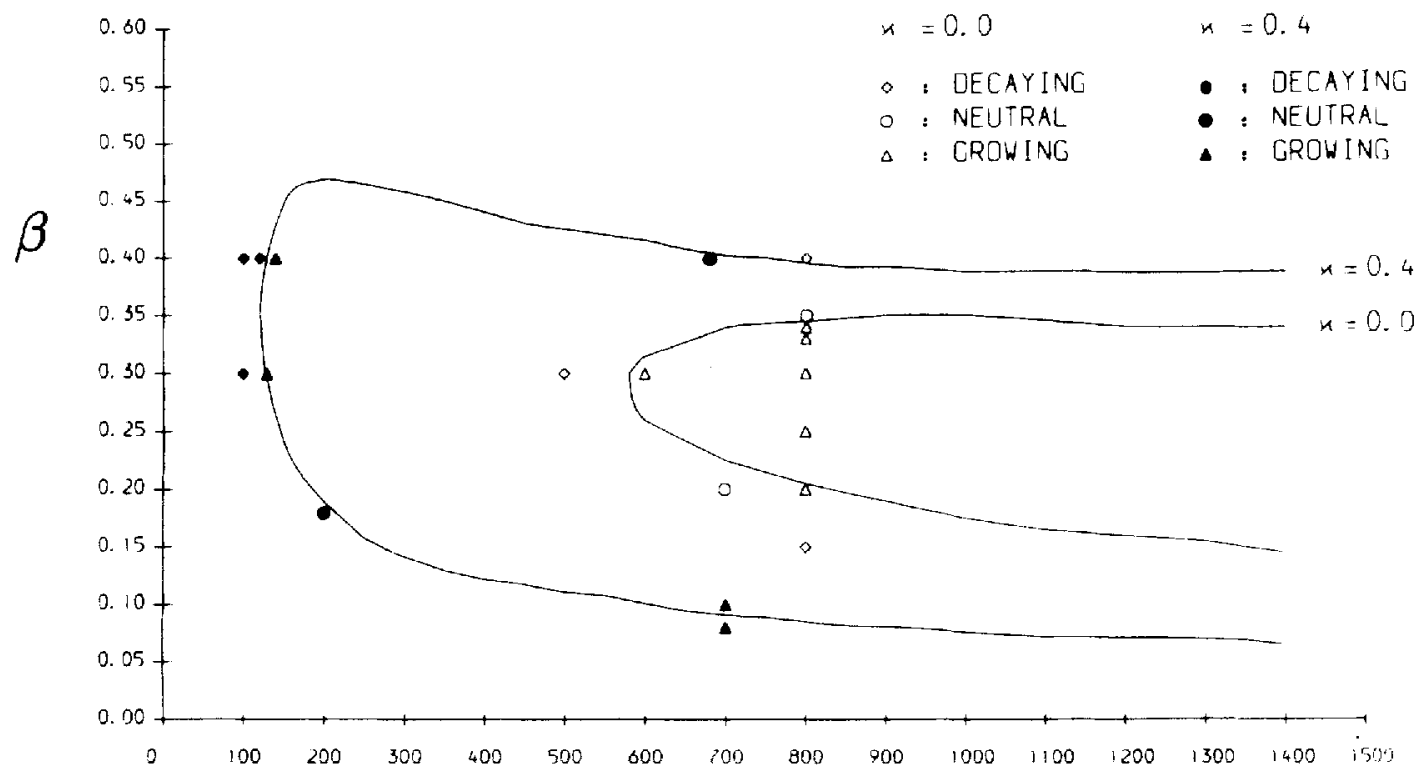

$R$

Figure 10. Comparison of results of Reference ? with our DNS

aforementioned problems, however, we compare DNS results presented earlier with results obtained using (well-established) linear stability theory tools in order to further validate our DNS approach.

\section{A TWO-DIMENSIONAL, LINEAR, TIME-PERIODIC SCHEME}

\subsection{Assumptions}

Time-periodic perturbations to the basic flow have also been considered (as assumption which is a standard investigation tool in linear stability theory). The basic flow is again taken to be the Hiemenz flow, while the assumptions of linearity together with the assumed $x$-variation of the streamwise components of velocity made in the time-marching case have been retained. Using the velocity-vorticity approach, we assume the following (temporal) structure of the perturbation flow quantities in physical space:

$$
\tilde{u}(y, z, t)=\hat{u}(y, z) \mathrm{e}^{\mathrm{i} \Omega t}+\text { c.c. }
$$

where tildes and hats again denote perturbations, $\Omega$ is a frequency and 'c.c.' denotes complex conjugate; a similar structure is assumed for the other velocity and for the vorticity components. Substituting the above forms into the basic set of equations (4) and (5), subtracting the Hiemenzflow-related terms and neglecting quadratic-in-perturbation quantities compared with the perturbations, we obtain equations of the form (in real space)

$$
\left(\frac{\partial^{2} \hat{u}}{\partial y^{2}}+\frac{\partial^{2} \hat{u}}{\partial z^{2}}-\frac{\partial \hat{\eta}}{\partial z}-\frac{\partial \hat{\zeta}}{\partial y}\right) \mathrm{e}^{\mathrm{i} \Omega t}+\text { c.c. }=0
$$


which may be simplified and Fourier transformed to obtain (in spectral space) equations of the form

$$
\frac{\partial^{2} \hat{u}^{*}}{\partial y^{2}}-\beta^{2} \hat{u}^{*}-\mathrm{i} \beta \hat{\eta}^{*}-\frac{\partial \hat{\zeta}^{*}}{\partial y}=0,
$$

similar equations holding for the other components of the velocity and vorticity fields. The boundary conditions for this system are no-slip for the $x$ - and $z$-components of the velocity and

$$
\hat{v}^{*}(y=0, \beta)=-\frac{1}{2} \mathrm{ie}^{-\beta^{2}}
$$

for the $y$-velocity component, while at the far field we expect all perturbations to go to zero. The specific form of boundary condition on the $y$-component of the velocity was chosen so as to correspond to the sinusoidal forcing of the time-marching scheme, ${ }^{*}$ thus enabling direct comparisons between the two schemes.

The system of equations consisting of (17) and the respective equations for the other two velocity and three vorticity components is solved in spectral space in a very similar manner as for the time-marching case. An important difference between this and the time-marching case is that the only non-zero components of the right-hand-side array $\mathbf{B}$ are now those associated with the boundary conditions. Of course, no time marching of the equations is required, because time in this time-periodic case only appears as a parameter. Once the system of equations has been solved, the equations of the generic form (15) are used to construct the field of perturbations in real space. Finally, this field is Fourier transformed from real into spectral space and a direct comparison of results obtained using the time-marching and time-periodic approaches is possible in both spaces.

\subsection{Results}

An extensive grid experimentation is also performed in this case to establish a grid independent solution. ${ }^{20}$ The optimum grid, again balancing resolution and memory requirements, is found to be that of the time-marching case. In performing the comparisons between the time-marching and time-periodic schemes, a relatively small time step $\Delta t=\pi / 64$ was chosen in order to give the former a smooth start and, for consistency, the sinusoidal wall forcing was chosen for the time-marching runs. The time-marching scheme was then run until it produced a transient-free solution. The elimination of transients was determined to have occurred by obtaining the solution at any time $t_{0}$ and comparing it with the solution at $t=t_{0}+2 \pi$. It was found to be necessary for time to grow as large as $t=10 \pi$ for the time-marching scheme to produce the results presented (although this value is Reynods-number- and $\Omega$-dependent).

The time-periodic solution at $t=\pi / 64$ was compared with the time-marching solution at $t=10 \pi+\pi / 64$. A representative result obtained in real space is shown in Figure 11 . One may readily notice the overall good agreement in the upstream as well as in the near-the-origin $(z=0)$ $z$-region. Further downstream the time-marching scheme shows oscillations which are not present in the time-periodic one. These are due to the transient behaviour to which we referred earlier and may be eliminated completely by time marching the solution even further, as results obtained but not presented here indicate.

The neutral curve for $\kappa=0$ shown in Figure 10 was again reproduced, this time in a spotcheck manner by appropriately choosing values for $(R, \beta)$ to correspond to those indicated in

$* \sin (\omega t)=-\frac{1}{2} \mathrm{ee}^{i \Omega t}+\mathrm{c.c}$. 


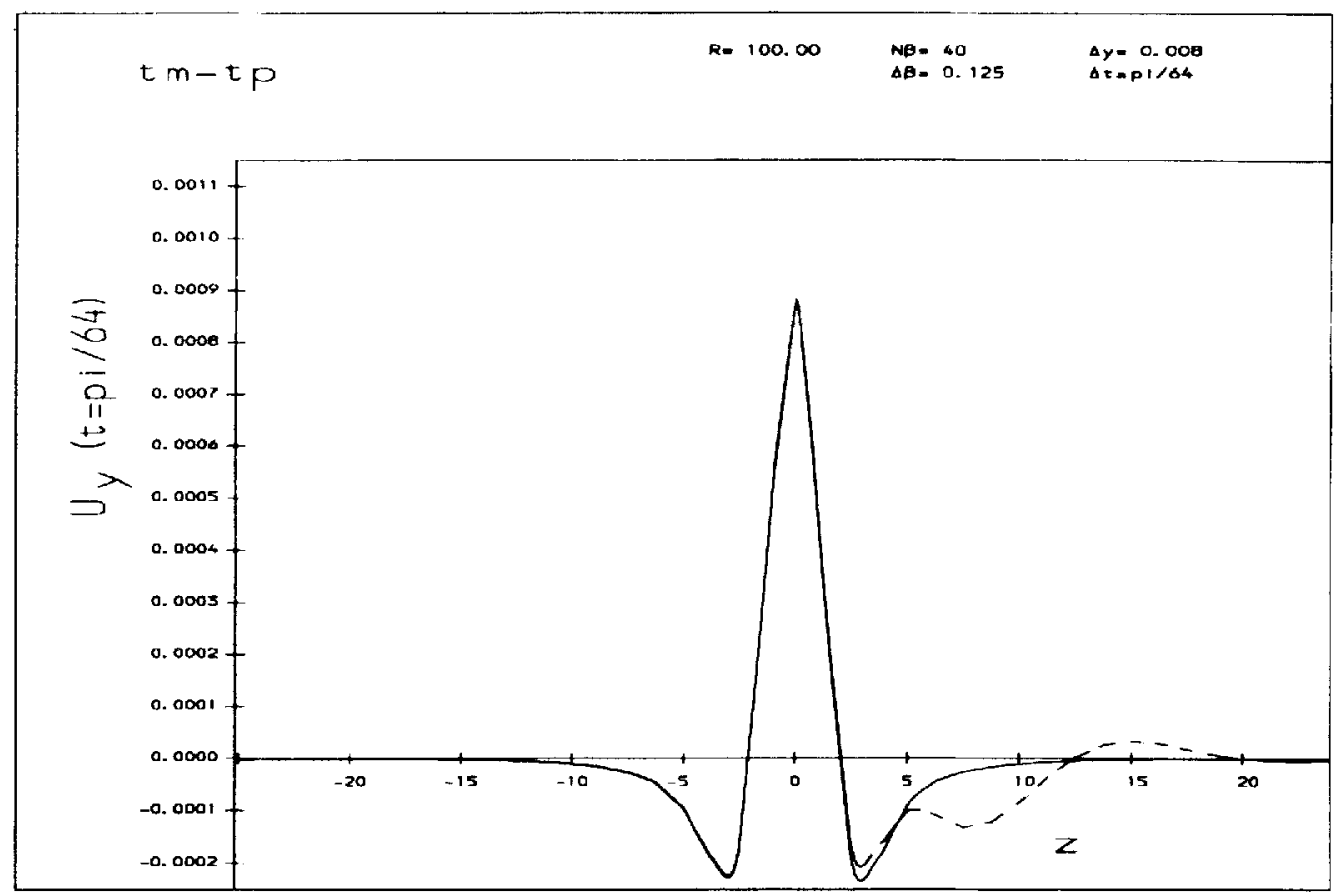

Figure 11. Representative comparison between time-marching $(--)$ and time-periodic $\left(\quad . \quad\right.$ results: $\hat{u}_{y}$

Reference 7. Again, the choice of the above parameters determined whether the particular flow was linearly stable or unstable, in a manner totally consistent with the findings of the previous section as well as those of Reference 7 .

As stated already, we want to adhere to our initial value problem approach, the results of which can only be trusted once the transients originally present in the solution have been eliminated. Additionally to building confidence in our codes by reproducing results of the time-marching approach such as neutral curve and growth rates, this time-periodic scheme served to provide us with a good estimate of the time required to elapse before the transient behaviour in the linear regime has subsided.

\section{CONCLUSIONS AND FUTURE EXTENSIONS}

A numerical study has been performed in order to assess the feasibility of a direct numerical simulation in studying the stability of LEBL flow. The main conclusion of this work is that such a study is indeed possible using an initial value problem approach, this being the novel element in our work compared with older theoretical studies based on normal mode analysis.

The linear regime of perturbations introduced in the infinite swept attachment line boundary layer flow was re-examined by our method and our findings were consistent with known results obtained by combination of asymptotic analysis and computation, solution of the eigenvalue problem or direct simulations. The gap between these and the experimental results, however, remains, thus motivating further study.

One of the requirements of the numerical scheme used was its potential to extend the present study to three-dimensional perturbations. Indeed, by introducing appropriate forms of perturba- 
tions and extending the spectral scheme used to three dimensions, one may study the stability of the LEBL flow at stations off the attachment line (in its vicinity but not too far off, where the cross-flow becomes progressively more dominant) using essentially the same numerical method. This idea was inherent in the choice of the velocity-vorticity formulation and work on the three-dimensional linear regime is currently under way.

Finally, with the gap between theoretical and experimental results persisting, work on experimental validation is being considered to be performed at the Goldstein Aeronautical Engineering Laboratory at Barton, Manchester in the continuing quest for reconciliation of results.

\section{ACKNOWLEDGEMENTS}

The able advice which Dr. P. W. Duck provided and the stimulating conversations with Professor D. I. A. Poll throughout the course of this work are gratefully acknowledged. The author wishes to thank the referees for their constructive comments which improved the original manuscript. Computations were performed on the Amdahl VP1100 and VP1200 supercomputers of the Manchester Computing Centre (MCC) under an SERC grant. The assistance of MCC staff with problems arising from the large-scale computations reported in this work is kindly appreciated.

\section{REFERENCES}

1. D. I. A. Poll, $A G A R D$ Rep. 709, VKI Lecture Series, Brussels, 1984, p. 5-1.

2. D. Arnal, $A G A R D$ Rep. 786 , VKI Lecture Series, Brussels, 1992, p. 5-1.

3. K. Hiemenz, Dingl. Polytechn. J., 326, 321 (1911).

4. H. Görtler, in H. Görtler and W. Tollmien (eds), 50 Jahre Grenzschichtforschung, Vieweg, Braunschweig, 1955 , p. 304.

5. G. Hämmerlin, in H. Görtler and W. Tollmien (eds), 50 Jahre Grenzschichtforschung, Vieweg, Braunschweig, 1955, p. 315.

6. P. R. Spalart, $A G A R D C P-438,1988$, p. 5-1.

7. P. Hall, M. R. Malik, and D. I. A. Poll, Proc. R. Soc. A, 395, 229 (1984).

8. P. Hall and M. R. Malik, J. Fluid Mech., 163, 257 (1986).

9. J. Jiménez, C. Martel, J. C. Agüi and J. A. Zufiria, ETSIA MF-903, 1990.

10. D. I. A. Poll, $A G A R D C P-22434,1977$, p. $21-1$.

11. D. I. A. Poll, Aeronaut. Q., 30, 607 (1979).

12. D. I. A. Poll, Proc. IUTAM Symp. on Laminar-Turbulent Transition, Stuttgart, Springer-Verlag, Berlin, 1980, pp. 253-262.

13. D. I. A. Poll, Aeronaut. Q., 34, 1 (1983).

14. D. I. A. Poll, J. Fluid Mech., 150, 329 (1985).

15. S. Goldstein, (ed.), Modern Developments in Fluid Dynamics, Dover, New York, 1965.

16. L. Howarth, $A R C$ R\&M 1632, 1935.

17. H. Schlichting, Boundary Layer Theory, McGraw-Hill, New York, 1979.

18. L. Rosenhead, Laminar Boundary Layers, Oxford University Press, Oxford, 1963.

19. V. Theofilis, M.Sc. Thesis, Department of Mathematics, University of Manchester, 1988.

20. V. Theofilis, Ph.D. Thesis, Department of Aeronautical Engineering, University of Manchester, 1991

21. S. C. R. Dennis, D. B. Ingham and R. N. Cook, J. Comput. Phys., 33, 325 (1979).

22. J. W. Cooley and J. W. Tuckey, Math. Comput., 19, 297 (1965).

23. P. W. Duck, J. Fluid Mech., 160, 465 (1985).

24. P. W. Duck and O. R. Burggraf, J. Fluid Mech., 162, 1 (1986).

25. O. R. Burggraf and P. W. Duck, in T. Cebeci (ed), Numerical and Physical Aspects of Aerodynamical Flows, SpringerVerlag, New York, 1981, p. 145.

26. J. Crank and P. Nicolson, Proc. Camb. Phil. Soc., 43, 50 (1946).

27. Numerical Algorithms Group (NAG), Scientific Library Mark 13, 1990.

28. A. Anscombe and L. N. Illingworth, ARC R\&M 2968, 1952.

29. H. Bippes, Proc. IU TAM Laminar-Turbulent Transition Symp. Toulouse, September 1989, Springer, 1990.

30. K. Brattkus and S. H. Davis, Q.J. Mech. Appl. Math., 44 pt. 1, 135 (1991).

31. C. Canuto, M. Y. Hussaini, A. Quarteroni and T. A. Zang, Spectral Methods in Fluid Dynamics, Springer, Berlin, 1987.

32. N. A. Cumpsty, and M. R. Head, Aeronaut. $Q ., 20,99$ (1969).

33. M. Gaster, Aeronaut. Q., 18, 165 (1967).

34. D. Gottlieb and S. A. Orszag, Numerical Analysis of Spectral Methods: Theory and Applications, CBMS-NSF Reg. Conf. Ser. Appl. Math., Vol. 26. SIAM, Philadelphia, PA, 1977. 
35. W. E. Gray, RAE Technical Memorandum Aero 255, 1952.

36. W. E. Gray, RAE TM Aero 256, 1952.

37. N. Gregory, J. T. Stuart and W. S. Walker, Philos. Trans. R. Soc. Lond. A, 248, 155 (1955).

38. P. Hall and S. Seddougui, NASA C R 181653, 1988.

39. C. C. Lin, Theory of Hydrodynamic Stability, Cambridge University Press, Cambridge, 1955.

40. M. J. Lyell and P. Huerre, J. Fluid Mech., 161, 295 (1985).

41. L. Mack, AGARD Rep. 709 , 1984, p. 3-1.

42. P. R. Owen and D. G. Randall, RAE TM Aero 330, 1953.

43. W. Pfenninger and J. W. Bacon, in C. S. Wells (ed.), Viscous Drag Reduction, Plenum, New York, 1969, p. 85.

44. D. I. A. Poll, in P. Stow (ed.), Computational Methods in Aeronautical Fluid Dynamics, Oxford University Press, Oxford, 1989 , p. 171.

45. H. L. Reed and W. S. Saric, Ann. Rev. Fluid Mech., 21, 235 (1989).

46. H. Schlichting, in S. Flügge (ed.), Handbuch der Physik, Vol. VIII/1, 1959, p. 351.

47. F. T. Smith, Proc. R. Soc. A, 366, 91 (1979).

48. F. T. Smith, Proc. R. Soc. A, 368, 573 (1979)

49. P. R. Spalart, NASA TM 88222, 1986.

50. H. B. Squire, $A R C 14,1952$, p. 991.

51. J. T. Stuart, $A R C$ 14, 1952, p. 991.

52. V. Theofilis, P. W. Duck and D. I. A. Poll, Proc. IU TAM Laminar-Turbulent Transition Symp., Toulouse, September 1989, Springer-Verlag, Berlin, 1990, p. 389.

53. V. Thcofilis, P. W. Duck and D. I. A. Poll, Lecture Notes in Physics, 371, 199 (1990).

54. W. Tollmien, 1. Mitteilung, Nach Ges. Wiss. Göttingen, Math.-Phys. Klasse, 21-44 (1929).

55. M. Van Dyke, Perturbation Methods in Fluid Mechanics, Academic, New York, 1964.

56. S. D. R. Wilson and I. Gladwell, J. Fluid Mech., 84, 517 (1978). 